\title{
Title: Anti-cancer activity of cuminum cyminum: A review.
}

\section{Authors: Chaudhari Nikhil Arun* ${ }^{1}$, Sathe Ninad ${ }^{2}$}

1. M.D. Scholar, Rasashastra and Bhaishajya Kalpana,

2. Prof. \& P.G. Guide, Mob.: 9820274619,; Email; ninadsathe@yahoo.com

\section{Rasashastra and Bhaishajya Kalpana,}

Dr. G. D. Pol Foundation's Y.M.T. Ayurvedic Medical College \& P.G. Institute, Kharghar, Navi Mumbai.

*Corresponding Author: Mob.: 9892220667, ; Email: nikhil967425663@ gmail.com

Abstract:

Jeeraka, commonly known as cumin (Cuminum cyminum Linn.) (Family: Umbelliferae), is a popular herbal plant in traditional ayurvedic medicine, which has since long used as antioxidant, gastric stimulant, antixfflatulence, anti-diarrheal, sprue, anthelmintic, galactagogue, diuretic, astringent, carminative. Th plant is pharmacologically and clinically evaluated for various activities like antiox dant, aphrodisiac, antimicrobial, hepato-protective, and anti-inflammatory. It is promulgated to possess a wide range of many phytochemical constituents. The current review is presented to give a comprehensive account of all anticancer activities of cumin. It will be helpful to create interest towards cumin and may be useful in developing new and economical anti-cancer formulations with more therapeutic value. The part used of the selected plant is seed.

As per retrospective review cumin seed, its essential oil, ethanoic extract and 1-(2-Ethyl, 6-Heptyl) Phenol (EHP), a biologically active compound formerly extracted by benzene are found having anti-cancer activity against 13 different cell lines of various origins and 6 animal models. Also, it can be used as chemopreventive ingredient in the herbal anticancer formulation. Total 8 research articles, peer viewed research papers, abstracts and classical texts were reviewed.

Keyword: Jeeraka, Cuminum cyminum Linn, cancer, 


\section{Introduction:}

According to GLOBOCAN ${ }^{\mathrm{i}}$ out of 14.1 million new cases diagnosed 8.2 million mortalities occurred due to cancer in 2012. The burden of the cancer cases will increase to 24 million new cases each year by 2035 . As per recent survey, more than $8,06,000$ cases are diagnosed and more than 0.3 million deaths/year in India due to cancer. According to National Cancer Registry Programme of the Indian Council of Medical Research (ICMR), more than 1300 Indians die every day due to cancer. Between 2012 and 2014, the mortality rate due to cancer increased by approximately 6\%. In 2012, there are $4,78,180$ deaths out of 29,34,314 cases reported. In 2013 there are 4,65,169 deaths out of $30,16,628$ cases. In 2014, 4,91,598 people died in 2014 out of 28,20,179 cases. This incidence rate has raised to $300 \%$ in past 4 decades and if th scenario remains same the burden present incidence rate will increase from $0.232 \%$ to $>1 \%$. ${ }^{\text {ii }}$

The role of carcinogenic agents in diets necessitates the perpetual search for natural anti-mutagens of promising anticancer therapeutics. Historically, natural products have been regarded as providing the primary leading compounds.

Cuminum cyminum is a small, slender annual herb, which is grown extensively in south-eastern Europe, north Africa bordering the Mediterranean-sea, in India and China. It is cultivated in almost all the states in India, chiefly including Uttar Pradesh, Punjab, Rajasthan, Gujarat and Maharashtra.

Its seeds have been used as an important condiment. According to Ayurvedic classics it is katu, ushna and pacifies deranged vata and effective as gastric stimulant, beneficial in abdominal lump, flatulence, diarrhea, sprue, hoarseness of voice and a strong anthelmintic. It is therapeutically used as an anti-diarrheal, galactagogue, diuretic, astringent, carminative. Paste of seeds on external application reduces pain and irritation due to worms in the abdomen. Its oil is useful in eczema.

Based on our past experience with the development of herbal drugs as anticancer agents from plants which are otherwise part of human diet and also documented to possess medicinal properties, we took up this plant to evaluate its anti-cancer effect, if any, based on its attributes bearing some similarities to the other plant sources with anti-cancer activity.

From Cuminum cyminum fruit rown in Delhi, 49 components were dentified in the essential oil constituents, which represented $99.78 \%$ of total detected constituents ${ }^{\mathrm{iii}}$ :-

1. Essential oil: monoterpene (79.61\%), sesquiterpene (2.66\%), aromatic $(16.55 \%)$ and aliphatic compounds $(0.66 \%)$.

2. Monoterpenes (34): fourteen hydrocarbons (41.28\%), twelve alcohols (5.76\%), six keto compounds (31.92\%), one aldehyde $(0.54 \%)$ and two esters $(0.11 \%)$.

3. The predominant monoterpene hydrocarbon was $\gamma$-terpinene $(23.22 \%)$ followed by $\alpha$-phellandrene $(12.01 \%)$, $\alpha$-pinene $(1.78 \%)$ and $\alpha$-terpinene $(1.24 \%)$.

4. Among twelve monoterpenic alcohols, p-menth-2-en-7-ol (3.48\%) was the major alcoholic constituent. 
5. The prominent monoterpenic ketone in the essential oil was transdihydrocarvone $(31.11 \%)$.

6. The sesquiterpenes identified in the oil were teresantalol $(2.62 \%)$ and karvaknol $(0.04 \%)$

7. The aromatic compounds detected were p-cymene (15.87\%), 8a-methyl octahydro-2 $(1 \mathrm{H})-$ naphthalenone, 2isopropyl-5-methyl phenol, p- cymen-7ol, o-cymen-5-ol, p-cymen-3-ol, 6-allyl4,5-dimethoxy-1,3-benzodioxole and 2,a,8,8-tetramethyl decahydrocyclopropanal naphthalene.

8. The aliphatic compounds included 1-(1, 2, 3-trimethyl-2- cyclopenten- 1-yl) ethanone, 3-isopropyl phenol, 2-methyl4-isopropyliden-cyclopentan-1-al, 1methyl-4-iso propyl-3- cyclohexen-1-ol, 2-isopropenyl-5-methyl-hex-4-enal, isopropyl cyclohex-1,3-dien-1-y methanol, 4-isopropyl-1-cyclohexencarbaldehyde, hexadecylene oxide and (3,4-dimethyl-2-oxo-cyclopenten-1-yl) acetic acid $^{(88)}$.

\section{ANTI-CANCER REVIEWS.}

A evaluated the cytotoxic activity of Cuminum cyminum ethanolic extract using in-vitro study. The anti-cancer activities of cumin seed, determined using SRB assay was found to be $25 \%, 61 \%$, $40 \%, 31 \%, 31 \%, 28 \%, 27 \%$ against SF295, Colon 502713, Colo-205, Hep-2, A549, OVCAR-5, PC-5 human cancer cell lines respectively. ${ }^{\text {iv }}$

\section{1-(2-Ethyl, 6-Heptyl) Phenol} (EHP), a biologically active compound formerly extracted by benzene from Cuminum cyminum (cumin), exhibited $0 \%$, $67 \%, 59 \%, 44 \%, 28 \%, 24 \%$ and negligible anti-tumor (cytotoxic) activity against the normal fibroblast (BHK), MCF7, HEPG2, HEP2, CACO2, HCT116 and HELA cell lines respectively. ${ }^{\mathrm{V}}$

The plant extract of Cuminum cyminum or its bioactive fraction can be extracted from its any plant parts and could be administered through oral, parenteral, nasal, inhalation including nebulizers, rectal, vaginal, transdermal and any other suitable routes.

The Bio-availability/Bio-efficacy Enhancing Activity effect of the extracts/fractions of Cuminum cyminum alone is selective in enhancing the bioavailability/bio-efficacy of Methotrexate, 5-fluotouracil, Doxorubicin, Cisplatin by $25,335,85,70 \%$ respectively. ${ }^{\mathrm{vi}}$

A known colon carcinogen, 1,2-dimethyl hydrazine (DMH) significantly increased beta-glucuronidase activity in the distal colon, distal intestine, liver, colon contents and mucinase activity in both the colon and fecal contents, when compared to that of control rats. Supplementation with cumin (Cuminum cyminum L., Apiaceae) in the presence of $\mathrm{DMH}$, showed more or less similar values as that of the control rats. The increase in beta- glucuronidase activity may increase the hydrolysis of glucuronide conjugates, liberating the toxins, while the increase in mucinase activity may enhance the hydrolysis of the protective mucins in the colon. Thus, cumin may protect the colon by decreasing the activity of betaglucuronidase and mucinase. Histopathological studies also showed lesser infiltration into the submucosa, 
fewer papillae and lesser changes in the cytoplasm of the cells in the colon in cumin group when compared to the DMH treated animals. ${ }^{\text {vii }}$

A study investigated the effect of cumin on colon cancer induced in rats by subcutaneous injection of a colon specific carcinogen, 1,2- dimethyl hydrazine $(\mathrm{DMH})$ at a dosage of $20 \mathrm{mg} / \mathrm{kg}$ of body weight (15 doses, at 1 week intervals). The rats were continued with the standard pellet diet and supplemented cumin seeds [1.25\% (wt/wt) mixed with the diet], throughout the experimental period (32 weeks including 2 weeks of acclimatization). After the total experimental period of 32 weeks the incidence and number of tumors in the colon were observed to be significantly higher in the rats administered DMH as compared with the cumin + DMH group No tumors were observed in the contro cumin $+\mathrm{DMH}$ groups. The results s that cumin suppresses colon carcinogenesis in the presence of the procarcinogen DMH. ${ }^{\text {viii }}$

The cumin essential oil exhibited a dose-dependent DPPH radical scavenging activity at $\mathrm{IC}_{50}$ concentration of $5.4 \mu \mathrm{g} / \mathrm{mL}$ and cytotoxic activity at $\mathrm{IC}_{50}$ concentration of $0.014 \mu \mathrm{L} / \mathrm{mL}$.

oil destructed Hela cells by $79 \%$ at a concentration of $0.1 \mu \mathrm{L} / \mathrm{mL}$. The antioxidant activity of cumin essential oil might contribute to its cytotoxic activity. Acute and sub-chronic toxicity was studied in a 30 days oral toxicity study by administration to Wistar rats of the essential oil. A $17.38 \%$ decrease in WBCs count, and $25.77 \%, 14.24 \%, 108.81 \%$ increase in hemoglobin concentration, hematocrit, and platelet count, respectively, were noted. LDL/HDL ratio was reduced to half, which adds to the nutritional effects of cumin. ${ }^{\text {ix }}$

In another study, cancer chemopreventive potentials of 2.5 and $5 \%$ doses of a cumin seed mixed diet were evaluated against benzo(a)pyrene [B(a)P] induced forestomach tumorigenesis and 3-methylcholanthrene (MCA)-induced uterine cervix tumorigenesis.

Cumin significantly inhibited stomach tumor burden (tumors per mouse). Tumor burden reduced from 7.33 +/- 2.10 in the B(a)P treated control group to $3.10+/-0.57$ by a $2.5 \%$ dose and 3.11 $+/-0.60$ by a $5 \%$ dose of cumin seeds in test groups.

Incidence of cervical carcinoma in MCA treated control group reduced from $6.67 \%$ to $27.27 \%$ by a diet of $5 \%$ cumin eeds and to $12.50 \%$ by a diet of $7.5 \%$ cumin seeds.

The effect of 2.5 and $5 \%$ cumin seed mixed diets was also examined on carcinogen/xenobiotic metabolizing phase I and phase II enzymes, antioxidant enzymes, glutathione content, lactate dehydrogenase (LDH) and lipid peroxidation in the liver of Swiss albino mice.

Levels of cytochrome P-450 and cytochrome b5 were significantly augmented by the $2.5 \%$ dose of cumin seed diet. The levels of cyt. P-450 reductase and cyt. b(5) reductase were increased by both doses of cumin. Among the phase II enzymes, glutathione S-transferase specific activity increased by the $5 \%$ dose, whereas that of 
DT-diaphorase increased significantly by both doses used.

In the antioxidant system, significant elevation of the specific activities of superoxide dismutase and catalase was observed with the $5 \%$ dose of cumin. The level of reduced glutathione measured as non-protein sulfhydryl content was elevated by both doses of cumin. Lipid peroxidation measured as formation of MDA production showed significant inhibition by both doses of cumin.

The results strongly suggest the cancer chemopreventive potentials of cumin seed and could be attributed to its ability to modulate carcinogen metabolism. ${ }^{\mathrm{x}}$

The anti-carcinogenic effect of cumin seed was studied in two models: th induction of squamous cell carcinomas the stomach by dietary benzo[a]pyrene $(\mathrm{B}[\mathrm{a}] \mathrm{P})$ in Swiss mice, and the induction of hepatomas by dietary Methyl- 4dimethylaminoazobenzene MeDAB in Wistar rats. 15 male mice in each group, 8 weeks old and weighing $23 \pm 2.1 \mathrm{~g}$, were used for all the experiments. The negative control group received standard powdered pellet diet while the positive control group received $\mathrm{B}[\mathrm{a}] \mathrm{P}$ alone in the diet and the test group received cumin seeds in the diet for 2 weeks, after which they also received $\mathrm{B}$ [a]P at a dose of $0.3 \mathrm{mg} / \mathrm{g}$ diet (in groundnut oil) three times a week for 8 weeks, mixed with the powdered diet. The incidence of tumours reduced from $77 \%$ in positive control group to $13 \%$ in cumin $+\quad$ benzo[a]pyrene administered group, i.e. inhibition by $83 \%$. 15 male Wistar rats in each group, 8weeks old and weighing $85-100 \mathrm{~g}$ were used for the experiments.
The negative control group received powdered diet, while the positive control group received MeDAB alone in the diet. The test group was fed cumin seed for 2 weeks after which they also received 3 MeDAB mixed in the diet at a concentration of $0.05 \%$ for 12 weeks.

The incidence of tumours reduced from 82 $\%$ in positive control group to $23 \%$ in cumin + Methyl- 4dimethylaminoazobenzene administered group. i.e. inhibition by $77 \%$ xi

\section{Conclusion:}

As per retrospective review cumin seed diet, its essential oil, ethanoic extract and 1-(2-Ethyl, 6-Heptyl) Phenol (EHP), a biologically active compound formerly xtracted by benzene are found having nti-cancer activity against 13 different eell lines of various origins and 6 animal models. Also, it can be used as chemopreventive ingredient in the herbal anti-cancer formulation. Total 8 research articles, peer viewed research papers, abstracts and classical texts were reviewed.

The selected herb Cuminum cyminum was reviewed for its seed diet, essential oil, ethanoic extract and 1-(2-Ethyl, 6-Heptyl) Phenol (EHP), a biologically active compound formerly extracted by benzene. It showed angiogenesis, antioxidant, radical scavenging, cytotoxicity, anti-mutagenic, mito-depressive, pro-apoptotic, anti-initiation, anti-promotion (delay the onset of tumor) and chemopreventive activity in a dose and time-dependent 
manner against various cell lines or animal models including SF-295, Colon 502713, Colo-205, Hep-2, A-549, OVCAR-5, PC5, normal fibroblast (BHK), MCF7, HEPG2, HEP2, CACO2, HCT116 and HELA human cancer cell lines; DMH induced colon carcinogenesis in wistar rats, benzo(a)pyrene $[\mathrm{B}(\mathrm{a}) \mathrm{P}]$ induced forestomach tumorigenesis in and 3-methylcholanthrene (MCA)-induced uterine cervix tumorigenesis in swiss albino mice, dietary benzo[a]pyrene $(\mathrm{B}[\mathrm{a}] \mathrm{P})$ induced squamous cell carcinomas of the stomach in Swiss mice and dietary
Methyl- 4-dimethylaminoazobenzene MeDAB induced hepatomas in Wistar rats. The probable mode of these anti-cancer activities in above cell line or animal models may be due to increased expression of Bax, Bid, Bad, Apaf-1, cytochrome c, caspase-9, -3 and PARP cleavage; inhibition of superoxide formation, lipid peroxidation, inducing increased detoxifying enzyme system glutathione S-transferase(GST) activity; modulatory effects on the hepatic levels of Cytochrome P- 450 (Cyt. P-450), Cytochrome b5 (Cyt. b5).

\section{References:}

i Cancer incidence and mortality worldwide: Sources, methods and major patterns in GLOBOCAN 2012, Int. J. Cancer: 136, E359-E386 (2015) VC 2014 UICC.

${ }^{i i}$ Principles of Internal Medicine, Harrison.

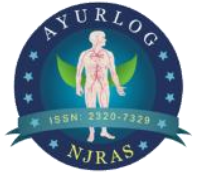

iii Chaudhary N, Husain SS and Ali M. Chemical composition and antimicrobial activity of cumin oil (Cuminum cyminum, Apiaceae). Journal of Pharmacy and Pharmaceutical Sciences 2014; 3(7): 14281441.

iv Ekta Prakash, Dwijendra Kumar Gupta. Cytotoxic Activity of Ethanolic Extract of Cuminum cyminum Linn Against Seven Human Cancer Cell Line. Universal Journal of Agricultural Research 2(1): $27-30,2014$

$\checkmark$ Mekawey, A. A. I.; Mokhtar, M. M.; Farrag, R. M. Antitumor and antibacterial activities of [1-(2-Ethyl, 6-Heptyl) Phenol] from Cuminum cyminum seeds. Journal of Applied Sciences Research 2009 No. November pp. 1881- 1888

\footnotetext{
${ }^{v i}$ Qazi et al. United States Patent. US 7,514,105B2. Apr. 7, 2009
} 
vii J Nalini N, Sabitha K, Viswanathan P, Menon VP. Influence of spices on the bacterial (enzyme) activity in experimental colon cancer. Ethnopharmacol. 1998 Aug;62(1):15-24.

viii Nalini N, Manju V, Menon VP. Effect of spices on lipid metabolism in 1,2-dimethylhydrazine induced rat colon carcinogenesis. J Med Food. 2006 Summer; 9(2): 237-45.

${ }^{i x}$ Tolou Allahghadri, Iraj Rasooli, Parviz Owlia, Mohammadreza Jalali Nadooshan, Tooba Ghazanfari, Massoud Taghizadeh, And Shakiba Darvish Alipoor Astaneh. Antimicrobial Property, Antioxidant Capacity, and Cytotoxicity of Essential Oil from Cumin Produced in Iran. JOURNAL OF FOOD SCIENCE-Vol. 75, Nr. 2, 2010

${ }^{x}$ Gagandeep, Dhanalakshmi S, Méndiz E, Rao AR, Kale RK. Chemopreventive effects of Cuminum cyminum in chemically induced forestomach ardorite cervix tumors in murine model systems. Nutr Cancer. 2003;47(2):171-80.

${ }^{x i}$ K. Aruna and V. M. Sivaramakrashnan Anticarcinogenic Effects Of Some Indian Plant Products Fd Chem. Toxic.Vol.30,No. 11,pp. 953-956,1992

Cite article:

Anti-cancer activity of cuminum cyminum: A review.

Chaudhari Nikhil Arun, Sathe Ninad

Ayurlog: National Journal of Research in Ayurved Science- 2018; (6)(4): 1-7 